


\title{
La importancia del cálculo integral en la ingeniería, caso específico: volumen de oleoductos ortogonales
}

\author{
Diego Carrillo Carvajal ${ }^{1}$, Karina Velásquez Flórez ${ }^{2}$, \\ Dairo Alfredo Causil Zúñiga ${ }^{3}$
}

\section{Resumen}

El diseño Volumen de oleoductos ortogonales pretende evidenciar cómo el cálculo integral es de gran utilidad en las ingenierías, destacando la posibilidad de encontrar el volumen de determinados objetos (Iglesias, Alonso, \& Gorina, 2017); en este caso, se trata del volumen común de dos cilindros del mismo radio que se cortan ortogonalmente, problema de suma importancia por su incidencia en los costos operacionales. Entre los materiales y recursos para la elaboración del diseño se encuentran tubos de papel higiénico, Colbón, silicona, tijeras, cartón cartulina, regla, cinta métrica, GeoGebra, entre otros. Este permite presentar el problema de volumen utilizando tanto el método teórico, con ayuda de la integral definida, como el método práctico con base en el software Geogebra de Geometría Dinámica (Villagrán, Cruz, Barahona-Avecilla , BarreraCárdenas, \& Insuasti-Castelo, 2018). Los resultados obtenidos son el diseño del recipiente en cartón cartulina con su respectivo volumen,

$1 \quad$ Magíster en Ciencias Matemáticas, Licenciado en Matemáticas. Jefe del Departamento de Ciencias Básicas de la Corporación Universitaria del Caribe-CECAR. Facultad de Ciencias Básicas, Ingenierías y Arquitectura. Líder de la colegiatura de Razonamiento Cuantitativo. Correo: diego.carrillo@cecar.edu.co; Orcid: https://orcid. org/0000-0001-5112-7771

2 Estudiante de Ingeniería Industrial. Corporación Universitaria del CaribeCECAR. Correo: karina.velasquez@cecar.edu.co; Orcid: https://orcid.org/0000-00023485-2952

3 Estudiante de doctorado en Ciencias Físicas, Magíster en Ciencias Físicas y Físico. Docente e investigador de la Corporación Universitaria del Caribe-CEGAR. Departamento de Ciencias Básicas, grupo de investigación "Ciencia, Entorno y Optimización”. Correo: dairo.causil@cecar.edu.co; Orcid: https://orcid.org0000-00026651-7913 
en el cual se evidencia de manera práctica que dicho sólido logra ajustarse con exactitud en la intersección de los oleoductos; lo anterior ayuda a señalar la importancia que tiene el aprendizaje del cálculo diferencial e integral para la ingeniería, al demostrar que con este conocimiento un ingeniero puede llegar a realizar diseños más precisos en áreas como la construcción de secciones de tuberías para oleoductos así como para cualquier proyecto que se pretenda realizar que necesite de un proceso de diseño.

Palabras clave: intersección, cilindro, integral, perpendicular, ortogonal.

\section{The importance of integral calculus in engineering, specific case: volume of orthogonal pipelines}

Abstract

The design "Volume of Orthogonal Oil Pipelines" aims to show how the integral calculation is very useful in engineering, where the possibility of finding the volume of certain objects stands out; in this case it is the common volume of two cylinders of the same radius that intersect orthogonally. This is a very important problem due to its impact on operating costs. Among the materials and resources for the elaboration of the design are tubes of toilet paper, glue, silicone, scissors, cardboard, ruler, measuring tape, GeoGebra, among others, which allows to present the volume problem using both the theoretical method with help of the definite integral as the practical method based on the Dynamic Geometry Geogebra software. The results obtained are the design of the cardboard cardboard container with its respective volume, in which it is evident in a practical way that said solid manages to fit exactly at the intersection of the pipelines. From the aforementioned design it can be concluded that it is vitally important to train engineers with the ability to appropriate the different concepts of calculation for solving problems, which are located mainly in fields related to their professional profile.

Keywords: intersection, cylinder, integral, perpendicular, orthogonal. 


\section{Introducción}

El hecho de enlazar dos o más partes en un oleoducto es un problema importante que si bien puede ser minimizado, ya sea controlando o tomando en cuenta factores topográficos en el trazado del mismo, no puede ser evitado; aun cuando se pueda minimizar el número de estos, siempre implicará costos adicionales. De aquí radica la importancia de estudiar los distintos tipos de empalme y la determinación de algunas características resultantes, entre las que destacan ciertas magnitudes asociadas a un cuerpo geométrico.

Para el caso de este trabajo, se plantea el estudio de la figura resultante del enlace de dos piezas cilíndricas del mismo diámetro en forma ortogonal, el cual es un problema que se aborda a partir del volumen común de dos cilindros que se cortan ortogonalmente. Este artículo de carácter pedagógicodivulgativo tiene como objeto de estudio un tema que se presenta con recurrencia en la disertación de diferentes profesores, donde se expone el análisis a fondo de la determinación de la magnitud mencionada a través del concepto de integral definida para su contextualización en aplicaciones de la ingeniería, en el cual es común comprobar la coincidencia de los resultados y así confirmar la exactitud de los conceptos del cálculo.

En ese sentido, el mencionado problema se encuentra planteado en diferentes libros como El cálculo de Louis Leithold (Leithold, 1994) y El cálculo integral de Jorge Sáenz (Saenz, 2009). Este último se toma como referencia principal para el diseño a realizar pues dicho libro tiene un enfoque para ciencias e ingeniería; en este documento se destaca la utilización del método teórico básico de las rebanadas, con el cual se obtiene una visión lo suficientemente clara para la elaboración del sólido resultante de la intersección de los oleoductos.

\section{Materiales y métodos}

El método teórico empleado es el de rebanadas, según el cual se corta el sólido en secciones transversales que son perpendiculares a uno de los ejes coordenados y, para el volumen, se calcula la integral definida del área de las secciones, donde los límites de integración están determinados por 
la oscilación de dichas secciones. Otro método teórico consiste en expresar el volumen a través de una integral doble, se debe tener mayor cuidado pues para este caso hay un ligero cambio dependiendo de la ubicación de los cilindros en el sistema tridimensional. Por otro lado, un método mucho más práctico y preciso que sirve para confirmar el resultado teórico es a través del software GeoGebra (Categoría:Tutoriales de Diseño-GeoGebra Manual, s.f.), además, es posible tener una mejor perspectiva del sólido y, de esa manera, poder elaborar con material reciclable la intersección de los cilindros, evidenciando experimentalmente que logra ajustarse con exactitud.

\section{Resultados y discusión}

El volumen deseado se puede obtener tanto con la integral definida como con la integral doble. El interés principal consiste en mostrar lo obtenido a partir de la primera opción. En primer lugar, se ubican en un sistema de coordenadas los dos cilindros del mismo radio r que se cortan ortogonalmente.

\section{Figura 1}

Cilindros ortogonales

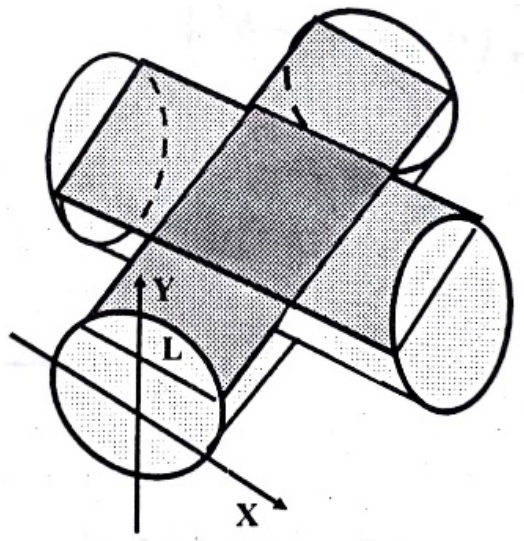

Nota. Fuente: tomado de Geogebra manual.

El problema consiste en hallar el volumen del solido encerrado por la intersección de dichos cilindros. Para ello se toma como referencia la circunferencia de radio $r$ que está sobre el plano $X Y$, de tal manera que al cortar el sólido con un plano perpendicular al eje $Y$ y a una altura de $y$ 
unidades, da como resultado una sección correspondiente a un cuadrado, cuyo lado se muestra a continuación (Tomás \& Sacta, 2020).

Figura 2

Circunferencia de referencia.

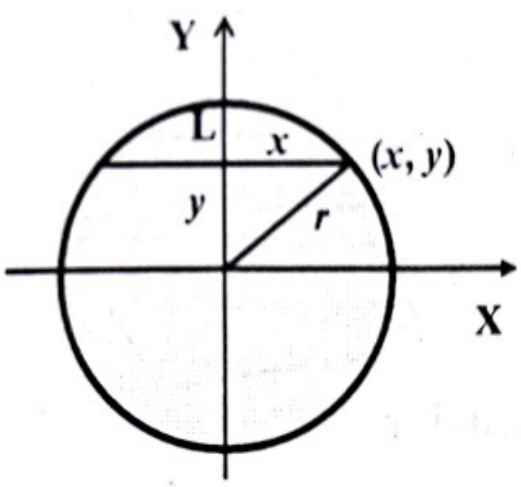

Nota. Fuente: tomado de (Saenz, 2009).

De acuerdo a lo anterior, el lado del cuadrado más grande está determinado por el diámetro de la circunferencia.

Figura 3

Sección transversal.

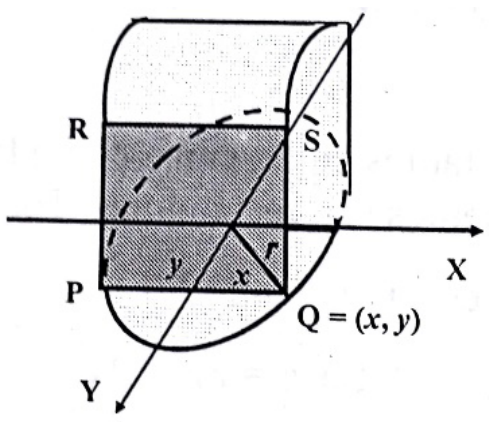

Nota. Fuente: tomado de Geogebra manual

En la imagen anterior se muestra el cuadrado cortando perpendicularmente al eje $Y$ a una altura de $y$ unidades, cuya longitud del lado es igual a $2 x$. Ahora bien, para obtener el sólido deseado se deben colocar los cuadrados concéntricamente, de tal forma que se abarque toda la circunferencia. 


\section{Figura 4}

Superficie de respuesta estimada.

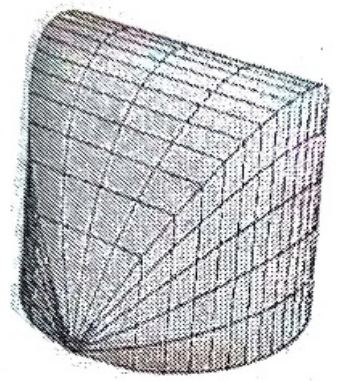

Nota. Fuente: tomado de (Saenz, 2009).

Finalmente, como el área de cada cuadrado está dada por $A(x)=$ $(2 x)^{2}=4 x^{2}=4\left(r^{2}-y^{2}\right)$, entonces, teniendo cuenta la simetría se obtiene (Briceño, 2010):

$$
V=\int_{-r}^{r} 4\left(r^{2}-y^{2}\right) d y=8\left[r^{2} y-\frac{y^{3}}{3}\right]_{0}^{r}=\frac{16}{3} r^{3}
$$

Por otro lado, se realiza el diseño del recipiente con material reciclable, donde se evidencia experimentalmente que dicho sólido logra ajustarse con exactitud en la intersección de los oleoductos. Los aspectos que entran en discusión hacen referencia principalmente al método teórico, pues se busca determinar el camino adecuado que ilustre propiedades suficientes para la elaboración del sólido en consideración. Lo primero que se destaca al examinar el método teórico dado por integrales dobles es la posibilidad de obtener ya sea la octava parte o la dieciseisava parte del volumen buscado, de acuerdo a la ubicación de los cilindros en el sistema tridimensional. Sin embargo, el aspecto que toma relevancia es el hecho de demostrar con total claridad el proceso que se debe llevar a cabo para obtener un objeto preciso, en el cual se representa el volumen común de los cilindros cortados ortogonalmente. 


\section{Conclusiones}

Se puede afirmar la importancia que tiene el aprendizaje del cálculo diferencial e integral para la ingeniería, al evidenciar que con este conocimiento un ingeniero puede llegar a realizar diseños más precisos en áreas como la construcción de secciones de tuberías para oleoductos, así como para cualquier proyecto que se pretenda realizar que necesite de un proceso de diseño. Asimismo, de la aplicación del cálculo diferencial e integral se obtiene una amplia información con la que se pueden diseñar modelos que simulen procesos reales ya sea a través de softwares o con maquetas interactivas como la que se realizó en el presente trabajo (Tejero Ruiz, 2015).

\section{Agradecimientos}

Agradecemos principalmente a quien fue nuestro profesor de Cálculo durante la licenciatura, por sus enseñanzas en la elaboración de diferentes sólidos; así como los profesores Javier Vélez y Orlando García, por su orientación en el diseño de la figura; de la misma forma, agradecemos a la estudiante de Cálculo Integral, Karina Velázquez, por su compromiso y colaboración con el diseño realizado.

\section{Referencias}

Briceño, F. J. (2010). Cálculo Diferencial e Integral-Volumen de un sólido. Retrieved from http://prof.usb.ve/bfeijoo/dat/MA1112/Guia09-I2010-A.pdf

Categoría:Tutoriales de Diseño-GeoGebra Manual. (n.d.). Retrieved from https://wiki.geogebra.org/es/Categor\%C3\%ADa:Tutoriales_de_Dise $\%$ C3\%Blo

Iglesias, N., Alonso, I., \& Gorina, A. (2017). El Cálculo Diferencial e Integral en las carreras de ciencias técnicas. Especificidades de su enseñanza. Maestro y Sociedad.

Leithold, L. (1994). El Cálculo. México: Fidencio Mata Conzáles.

Saenz, J. (2009). Cálculo Integral para Ciencias e Ingeniería. Barquisimeto.

Tejero Ruiz, J. F. (2015). EXPLORACIÓN DEL CÁLCULO INTEGRAL DESDE EL CONTEXTO DE LA GEOMETRÍA DINÁMICA. Bogotá.

Tomás, R. T., \& Sacta, T. M. (2020). GUÍA DIDÁCTICA PARA MEJORAR LA ENSEÑANZA DE LA INTEGRACIÓN MÚLTIPLE APLICADA 


\section{AL CÁLCULO DE ÁREA Y VOLUMEN DE SÓLIDOS CON APOYO DE RECURSOS EDUCATIVOS. Ecuador.}

Villagrán, W. J., Cruz, E. L., Barahona-Avecilla, F. R., Barrera-Cárdenas, O. B., \& Insuasti-Castelo, R. M. (2018). Utilización de GEOGEBRA como herramienta metodológica en la enseñanza de la geometría Analítica y su incidencia en el control del rendimiento académico de estudiantes del primer semestre de ingeniería. Dominio de las Ciencias, 4(4). 